\title{
SUPPLEMENTARY INFORMATION: HTMD: High-throughput molecular dynamics for molecular discovery
}

\author{
S. Doerr, ${ }^{1}$ M. J. Harvey, ${ }^{2}$ Frank Noé, ${ }^{3}$ and G. De Fabritiis ${ }^{4,1, *}$ \\ ${ }^{1}$ Computational Biophysics Laboratory (GRIB-IMIM), \\ Universitat Pompeu Fabra, Barcelona Biomedical Research Park (PRBB), \\ C/ Doctor Aiguader 88, 08003 Barcelona, Spain \\ ${ }^{2}$ Acellera, Barcelona Biomedical Research Park (PRBB), \\ C/ Doctor Aiguader 88, 08003 Barcelona, Spain \\ ${ }^{3}$ Department of Mathematics, Computer Science and Bioinformatics, Free University of Berlin, Berlin, Germany \\ ${ }^{4}$ Institució Catalana de Recerca i Estudis Avançats (ICREA), \\ Passeig Lluis Companys 23, Barcelona 08010, Spain
}

\section{ADAPTIVE EQUATIONS}

First, all currently available trajectories are projected onto a given metric space using any of the projection classes described in Table 1 of the main document. Then, the projected conformations are clustered using a clustering method into a set of $N_{k}$ clusters. The number of clusters, $N_{k}$, is determined by the amount of total conformations using the curve show in Figure S1. After clustering, a Markov model is constructed at a lag-time of 1 step and $N_{m}$ macrostates are obtained using the PCCA $+^{11}$ algorithm. Then, the number of conformations in each macrostate $M_{c}^{m}$ is determined by summing up the conformations in the clusters corresponding to each macrostate.

$$
M_{c}^{m}=\sum_{k=1}^{N_{k}} \mathbb{1}_{m}(k) C_{k}
$$

where $C_{k}$ indicates the number of confomations in cluster $k$ and $\mathbb{1}_{m}(k)$ is the indicator function which is 1 if cluster $k$ belongs to macrostate $m$ and 0 otherwise. Lastly, the counts of each macrostate are inverted and normalized, giving a probability distribution $p(m)$.

$$
\begin{gathered}
z_{m}=\frac{1}{M_{c}^{m}} \\
p(m)=\frac{z_{m}}{\sum_{m=1}^{N_{m}} z_{m}}
\end{gathered}
$$

We then define a multinomial distribution on the random variables $X_{m}$, with associated probabilities $p(m)$ with nmax - nrun trials giving us the number of conformations we should restart from each macrostate.

* Electronic address: gianni.defabritiis@upf.edu

${ }^{1}$ P. Deuflhard and M. Weber, Linear Algebra Appl. 398, 161 (2005), ISSN 0024-3795, URL http://www.sciencedirect.com/ science/article/pii/S0024379504004689 


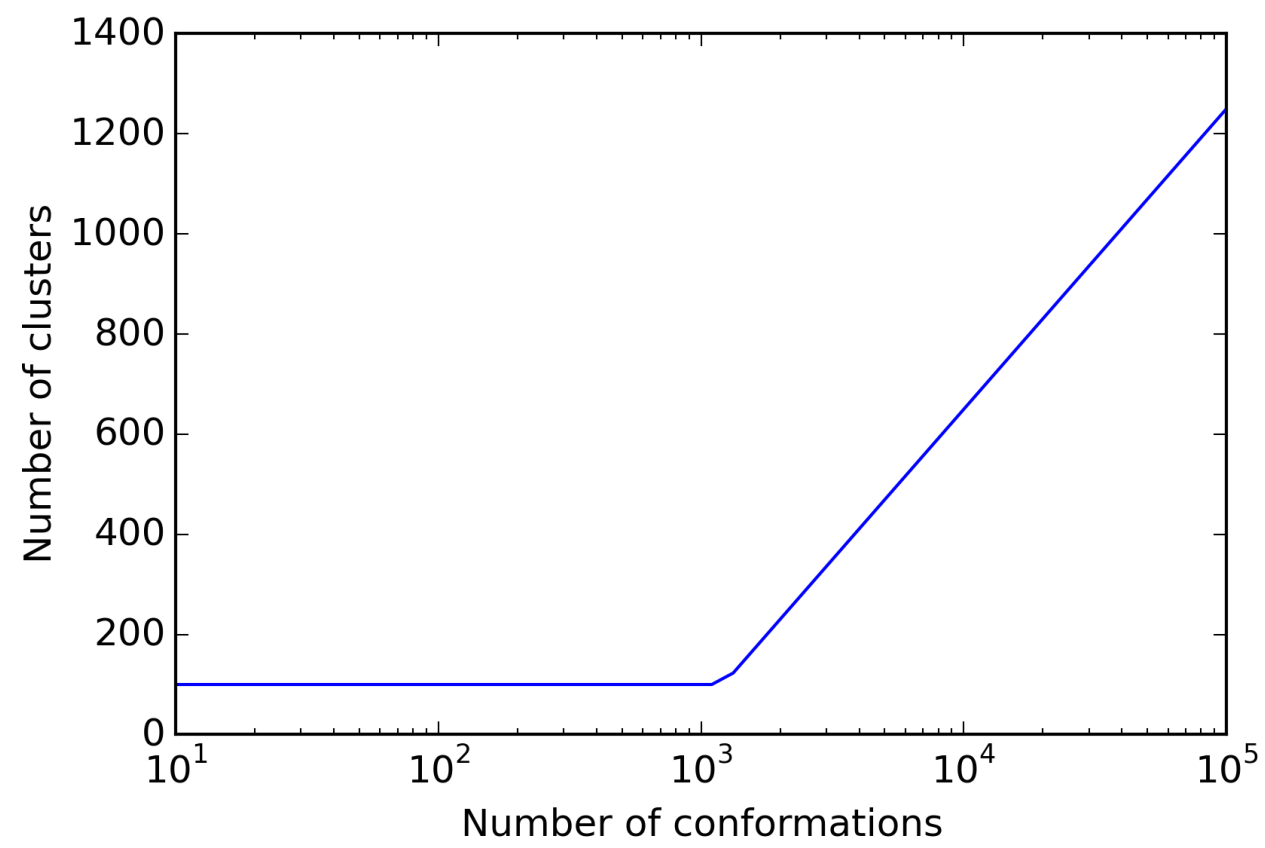

FIG. S1: Number of clusters used in the Markov models constructed during adaptive sampling. The number of clusters scales logarithmically with the number of conformations available.

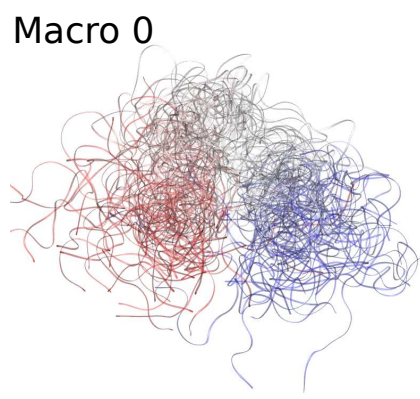

Macro 2

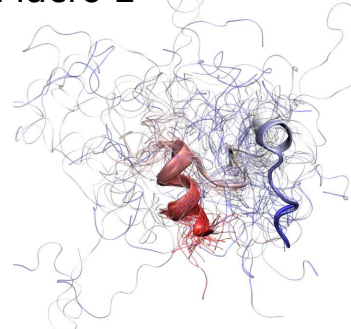

Macro 1

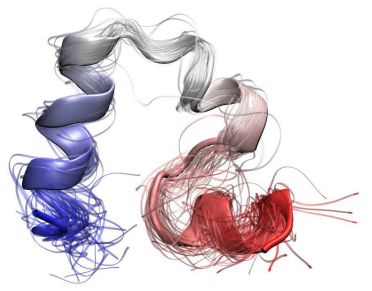

Macro 3

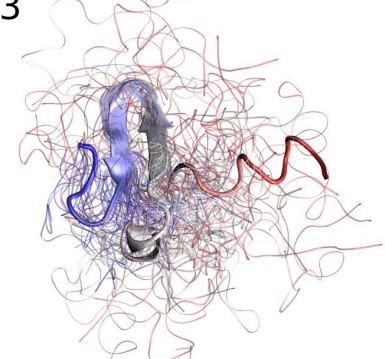

FIG. S2: The 4 macrostates produced by the Markov state model of Villin. Macrostate 0 is the unfolded state (containing various short-lived secondary structures), macrostate 1 corresponds to the folded structure, macrostate 2 corresponds to the red helix forming and macrostate 4 corresponds to a beta sheet formation. 